\title{
Epidemiological characteristics of female population with chronic spinal cord injury \\ Ana Esclarín ${ }^{1}$, Mónica Alcobendas ${ }^{1}$, Carlos Vicario ${ }^{2}$, Ramiro Palazon ${ }^{1}$, Rosa María Casado ${ }^{1}$, Silvia Ceruelo \\ ${ }^{1}$ Department of Physical Medicine and Rehabilittion. Hospital Nacional Paraplejicos. Toledo. Spain \\ ${ }^{2}$ Department of Orthopaedic Surgery. Hospital Nuestra Senora del Prado. Talavera de la reina, Spain.
}

\section{Introduction.-}

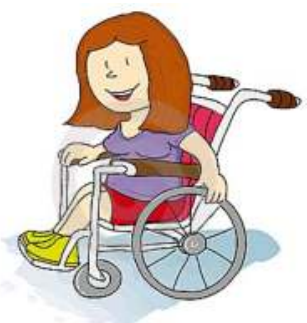

Patients gender can drive to long-term differences in evolution of spinal cord injury (SCI). Specific studies about SCI in women are necessary because genetic and epidemiological differences such as etiology, medication, osteoporosis, exercise, assistance, reproduction, etc can be very important

Purpose.-

To know long-term complications and epidemiological and functional characteristics in women with SCI.

\section{Method.-}

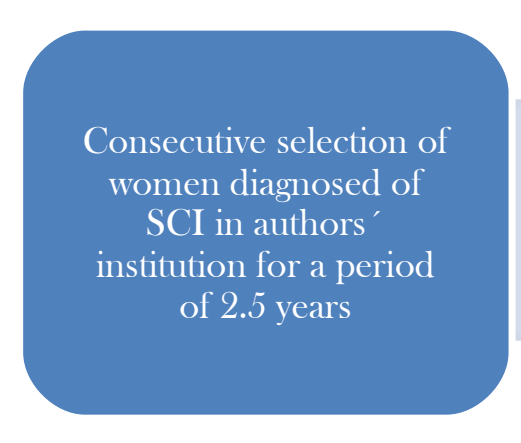

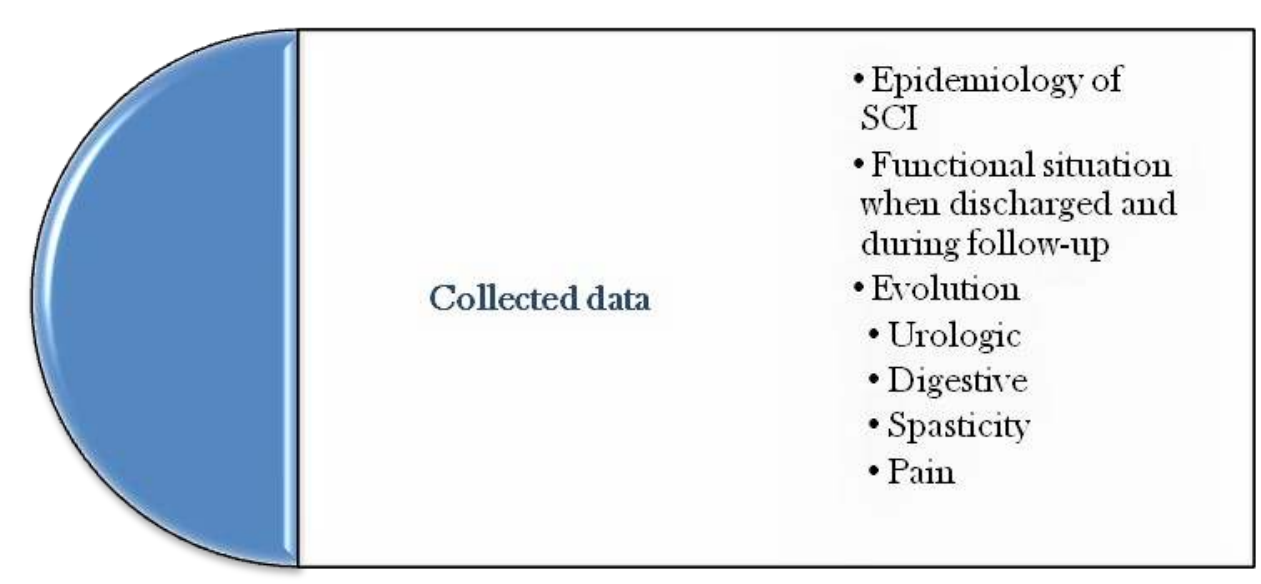

Statistical analysis: Frequencies, Means, SD, Paired mean comparison, $\mathbf{X}^{2} \mathrm{p}<0.05$

\section{Results.-}

More than 1 year of evolution

Consent to participate in this study
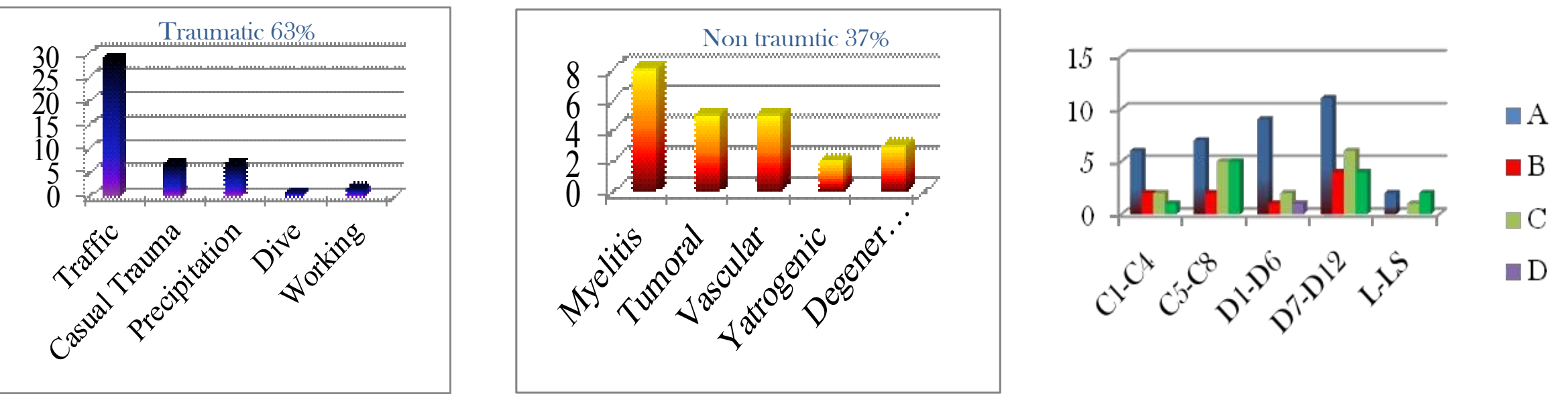

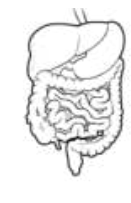

Bowel habit more frequent in 481 Use of laxative: $84 \%$ 16\% complications: $58 \%$ haemorrhoid (not related with age or time of evolution) $\underline{\text { Pain }}$

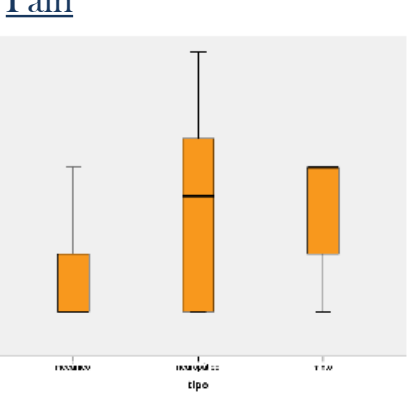

In the level or bellow SCI: $62 \%$

$58 \%$ Required treatment (72\% Gabapentine/Pregabaline) Higher frequency of pain in thoracic SCI was found p 0,05 No relation with age or time of evolution

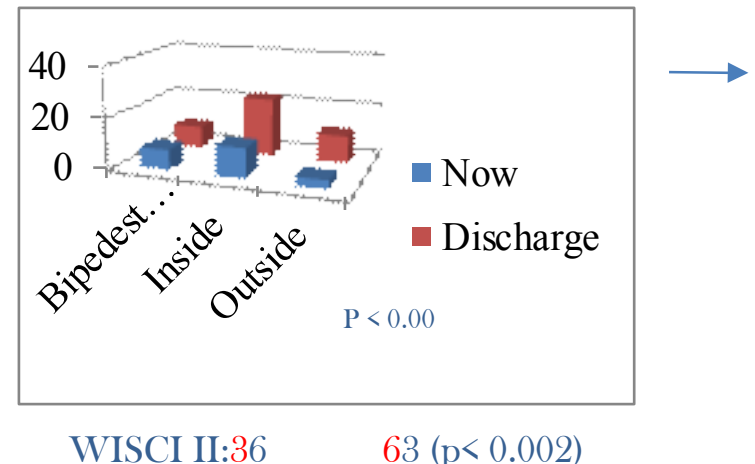
cation: trochanter $11 \%$, sacrum $5 \%$, isquion $4 \%$ Related with time of evolution $(\mathrm{p}<0.002)$

Conservative treatment: $84 \%$
$20 \%$ pressure ulcers

\section{$\pi^{2}$}

Upper motor neurone lesion: $65 \%$ $46 \%$ Pharmacological treatment Modified Ashworth: 2 (SD1) No relation with age or time of evolution

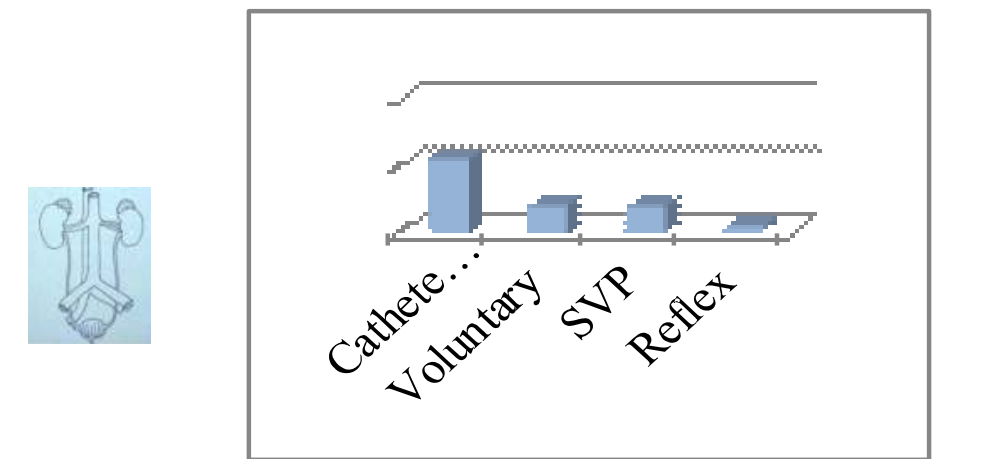

$20 \%$ need some kind of treatment ( $85 \%$ anticolinergic). Catheterization and voluntary were related with lower risk of UTI $(\mathrm{p}<0.001)$

Urinary tract infection: 1.5 per year

$5 \%$ Vesicoureteral reflux
Myelitis was the most frequent medical cause (48\%). Thoracic and complete SCI were the most frequent. Intermittent catheterization urination in $41 \%$, and urinary tract infection in nearly every case with a mean of 1.5 episodes per year were the most common urinary complications. Other common complications were: urethra-bladder reflux (16\%), haemorrhoid (30\%), pressure ulcers $(30 \%), 65 \%$ of upper motor neuron injuries developed Ashwoth 2 spasticity and 46\% of them required medical treatment. Pain was reported in 77\% with a mean VAS of 3, it was neuropathic below SCI level in $46 \%$ and was related with increasing age. When discharged, 43 patients were able for bipedestation, but 13 of them gave up. A significative decrease during follow-up in WISCI and FIM were found.

\section{Conclusions.-}

Actually, less than $25 \%$ of chronic SCI patients reviewed in author's institution are women Traumatic and complete lesions are more frequent.

The only complication related with time of evolution are pressure ulcers.

Permanent urinary catheter has been related with a higher risk of urinary tract infection.

Significative pain is common, specially neuropathic pain, and it is more frequent in thoracic SCI.

Only half of the patients with spasticity require pharmacological treatment.

Gait ability and functional independence are impaired in longer evolution patients. 